Methods Borderline advanced malignancy patient underwent diagnostic workup and was found to have adenocarcinoma uncinate. He underwent palliative double bypass and NACT. Post-NACT reassessment was done, which showed a resectable uncinate mass with the dilated pancreatic duct. Curative resection -Whipple's pancreaticioduodenectomy was done. Biopsy showed -complete response.

The postsurgery-controlled pancreatic fistula was managed conservatively, but afferent loop of GJ and leakage developed which was treated initially with prolonged conservative treatment. After the failure of conservative TT it was operated and adhesions lysed with jejunojejunal bypass was done. Still patient had $10 \mathrm{ml}$ leakage from drain site pancreaticojejunostomy or gastrojejunostomy leak. Again conservative TTT was done but failed. Fistula was located by percutaneous tube and fibrin glue injection was done to heal the fistula tract which succeeded, but later again, $5 \mathrm{ml}$ occasional discharge lead to exploration and fistula finally healed. The patient remained in follow up to $2.5 \mathrm{yrs}$ since diagnosis and 1.5 yrs since the curative surgery, without any recurrence.

Results The patient remained disease-free for 1.5 yrs post curative surgery and 2.5 yrs since diagnosis.

The gastrojejunaula healed after multiple interventions.

Conclusions The multispecialty approach gives the best results in borderline advanced uncinate malignancy

Persistence leads to the cure of any GI surgical issues in patients

Prolonged survival can be expected in curative surgery of post-NACT carcinoma uncinate

A complete response can be seen in uncinate malignancy cases.

\section{IDDF2020-ABS-0193 UNDIFFERENTIATED-TYPE-PREDOMINANT MIXED-TYPE IS MORE AGGRESSIVE THAN PURE UNDIFFERENTIATED-TYPE IN EARLY GASTRIC CANCER: A META-ANALYSIS}

Peng Yang*. Changzhou No.2 People's Hospital, China

\subsection{6/gutjpl-2020-IDDF.115}

Background Previous studies show that Differentiated-type-predominant Mixed-type(MD) Early Gastric Cancer(EGC) shows more aggressive behavior than Pure Differentiated-type(PD) EGC. However, the biological behavior of Undifferentiatedtype-predominant Mixed-type(MU) EGC and Pure Undifferentiated-type(PU) EGC are controversial. The aims of this metaanalysis were to compare the biological behaviour between MU EGC and PU EGC.

Methods We systematically searched PubMed and EmBase for relevant studies published up to June 2020. Eligible data were extracted, the pooled results were expressed with odds ratios (ORs) and 95\% confidence intervals (95\% CIs) using Stata software, version 15.1 .

Results In total, 8 studies were included in this analysis. MU EGC had a significantly higher lymph node metastasis(LNM) risk (OR, 2.66; 95\% CI, 2.24-3.18), submucosal invasion risk (OR, 2.44; 95\% CI, 1.55-3.84), and lymphovascular invasion risk (OR, 2.66; 95\% CI, 2.08-3.41) compared with PU EGC. Otherwise, stratified by country, a significantly higher lymph node metastasis(LNM) risk (OR, 2.56; 95\%
CI, 2.15-3.05), submucosal invasion risk (OR, 3.72; 95\% CI, 1.08-12.78), and lymphovascular invasion risk (OR, 2.77; 95\% CI, 2.14-3.58) correlative to MU EGC were found in Japan studies.

Conclusions Our study identifies MU EGC had an increased risk of submucosal invasion, lymphovascular invasion and LNM compared with PU EGC, which indicated that we should pay more attention to patients with MU EGC in clinical management.

\section{IDDF2020-ABS-0194 TIMING OF ENDOSCOPY FOR ACUTE UPPER GASTROINTESTINAL BLEEDING: A TERRITORY-WIDE COHORT STUDY}

${ }^{1}$ Cosmos Liutao Guo*, 'Sunny Hei Wong, 'Louis Ho Shing Lau, 'Rashid Nok Shun Lui, ${ }^{1}$ Joyce Wing Yan Mak, ${ }^{1}$ Raymond Shing Yan Tang, ${ }^{2}$ William Ka Kei Wu, ${ }^{1}$ Grace Lai Hung Wong, ${ }^{1}$ Francis Ka Leung Chan, ${ }^{3}$ James Yun Wong Lau, ${ }^{1} J$ oseph Jao-yiu Sung. ${ }^{1}$ Department of Medicine and Therapeutics, Faculty of Medicine, the Chinese University of Hong Kong, Hong Kong; 'Department of Anaesthesia and Intensive Care, Faculty of Medicine, The Chinese University of Hong Kong, Hong Kong; ${ }^{3}$ Department of Surgery, Faculty of Medicine, the Chinese University of Hong Kong, Hong Kong

\subsection{6/gutjpl-2020-IDDF.116}

Background Whilst it is commonly recommended that patients presenting with acute upper gastrointestinal bleeding (AUGIB) should receive endoscopic evaluation within 24 hours, the optimal timing is yet to be established. We aimed to assess, for AUGIB patients, whether endoscopy timing after hospital admission would significantly affect clinical outcomes.

Methods We conducted a retrospective, territory-wide, cohort study with clinical data extracted from the Hong Kong Hospital Authority (HA) Clinical Data Analysis and Reporting System (CDARS). Patients of age 18 and above that (a) presented with AUGIB to the public hospital between 2013-2019 and (b) received therapeutic endoscopy within 48 hours ( $\mathrm{n}=$ 6,474 ) were recruited.

Patients were classified into 3 groups based on endoscopic timing after admission: urgent $(t \leq 6)$, early $(6<\mathrm{t} \leq 24)$ and late $(24<\mathrm{t} \leq 48)$. Important baseline characteristics (e.g. blood tests and comorbidities) were balanced with inverse probability of treatment weighting (IPTW). After balancing, the 30-day all-cause mortality, 30-day repeated therapeutic endoscopy rate, 30-day ICU admission rate and other endpoints were compared. We hypothesised that, amongst the three groups, early timing would be associated with the best clinical outcomes. Subgroup analysis was performed based on comorbidity burden and aetiology of bleeding. Sensitivity analysis was performed.

Results With IPTW, differences in patient characteristics across 3 groups were well balanced. Results showed that urgent (n $=1,008)$ and late endoscopy $(n=1,601)$ had worse outcomes compared to early endoscopy $(\mathrm{n}=3,865)$, with higher 30-day mortality, repeat endoscopy rates and ICU admission rates.

Conclusions Compared to urgent and late endoscopy, early endoscopy timing was significantly associated with superior outcomes. This supports the notion that AUGIB patients should receive endoscopy within 24 hours, but also emphasises the importance of adequate resuscitation, medical optimisation and pharmacotherapy before rushing patients to endoscopy. 